\section{Cahiers de littérature orale}

$83 \mid 2018$

Geneviève Calame-Griaule

\title{
La séance, unité pertinente d'analyse en littérature orale
}

The Collective Performance as a Relevant Level for Studying Oral Literature

Jean Derive

\section{(2) OpenEdition}

\section{Journals}

Édition électronique

URL : https://journals.openedition.org/clo/4654

DOI : $10.4000 /$ clo.4654

ISSN : 2266-1816

Éditeur

INALCO

Édition imprimée

Date de publication : 21 juin 2018

Pagination : 55-77

ISBN : 9782858313143

ISSN : 0396-891X

Référence électronique

Jean Derive, «La séance, unité pertinente d'analyse en littérature orale », Cahiers de littérature orale [En ligne], 83 | 2018, mis en ligne le 20 juin 2019, consulté le 01 juillet 2021. URL : http://

journals.openedition.org/clo/4654; DOI : https://doi.org/10.4000/clo.4654

\section{(C) $(1) \otimes$}

Cahiers de littérature orale est mis à disposition selon les termes de la Licence Creative Commons Attribution - Pas d'Utilisation Commerciale 4.0 International. 


\title{
La séance, unité pertinente d'analyse en littérature orale
}

\author{
Jean Derive \\ Université de Savoie - LLACAN
}

Geneviève Calame-Griaule, dès ses premiers séjours chez les Dogon, a eu l'intuition de l'intérêt capital, pour étudier les pratiques langagières d'une société, d'une approche ethnolinguistique qu'elle n'a par la suite cessé d'approfondir, devenant même, pour la littérature orale africaine, l'un des principaux théoriciens français de cette discipline ${ }^{1}$. Un tel positionnement théorique implique certaines exigences au double plan de la collecte et de l'analyse.

Au niveau de la collecte, selon l'approche ethnolinguistique, les œuvres orales doivent autant que possible être recueillies dans des conditions « naturelles » de production, c'est-à-dire lorsqu'elles adviennent spontanément dans la vie culturelle de la société, sans sollicitation extérieure. Le respect de cette condition permet, plus sûrement qu'une enquête, d'en établir tous les paramètres du contexte d'énonciation : statut des co-énonciateurs et de tous les partenaires de la performance, temps, lieu et modalités d'exécution.

Au niveau de l'analyse, la mise en regard de tous ces facteurs avec l'énoncé permet de mieux saisir la fonction sociale de l'événement culturel étudié. Au-delà de son signifié immédiat, celui-ci est alors en effet pris en compte dans toutes ses composantes, le verbal n'occupant plus nécessairement la place centrale pour établir le sens de ce procès énonciatif actualisé dans une performance donnée.

1. Cf. Calame-Griaule, 1970, 1977 et 2001. 
Cet intérêt central porté à la performance a d'autres incidences. Il permet parfois de modifier le niveau de ce qu'il est pertinent de prendre pour objet d'étude. En régime oral, quelle que soit la culture considérée, il est d'usage que, pour un certain nombre de genres, en général d'un volume assez modeste, soit décliné en série tout ou partie de leur répertoire (par exemple : contes, chants, devinettes...) au cours d'une séance, cérémonielle ou non.

La plupart du temps, dans les publications consacrées à la littérature orale, les unités énonciatives qui font l'objet d'une analyse sont précisément ces « œuvres » individuelles relevant d'un genre donné. Certes, pour les genres plus ou moins brefs tels que les contes, les chants, les proverbes et devinettes, ces items sont généralement regroupés en recueil et, dans certains cas, c'est l'ensemble de ce recueil qui est analysé pour mettre en évidence des traits stylistiques ou des récurrences de motifs propres au genre. Toutefois, la plupart du temps, ces corpus ont été constitués sur des bases formelles ou thématiques, plus rarement énonciatives (chants d'hommes ou de femmes, par exemple). Mais il est extrêmement rare que les œuvres soient présentées dans l'ordre sériel où elles ont été exécutées au cours d'une performance et que cette succession soit considérée comme un facteur pertinent. Nous voudrions mettre en évidence que la prise en compte de l'intégralité d'une séance où se déroule une série d'œuvres individuelles est capitale pour saisir pleinement la signification culturelle de l'événement énonciatif.

Pour illustrer cette thèse, nous allons nous appuyer sur quelques exemples empruntés à certains genres de la littérature orale des Dioula de Kong où j'ai précisément recueilli un corpus varié, dans le cadre de séances produites in situ, en dehors de mon initiative.

\section{Le cas des genres régis par un rituel}

Dans la société dioula, il existe un certain nombre de genres chantés dont le répertoire d'œuvres est exécuté suivant un déroulement fixé à l'avance et calqué sur un rituel immuable. Il en va ainsi des différents types de chants de masque [dò dònkiri] qu'on retrouve interprétés dans un ordre quasiment identique d'une performance à une autre. Ce déroulement fixe, lors de la sortie du masque, s'explique par le fait que celui-ci suit un itinéraire programmé qui le mène dans différents quartiers [kábila] de Kong. Et à chaque kàbila correspond une série de chants spécifiques qui dépend de la nature de la relation que le masque entretient avec les familles de ce quartier auxquelles il s'adresse successivement. Il va de soi que c'est alors la séance dans son ensemble, suivant son déroulement spécifique, 
qui fait sens dans la mesure où elle éclaire le signifié de chaque chant particulier qui, sans cette clé, demeurerait souvent obscur.

Suivant le même principe, la succession des chants nuptiaux de déploration [kónyın kòmbo dj̀nkiri], exécutés par la mariée à l'occasion des cérémonies nuptiales, est déterminée par le parcours qu'elle accomplit pour faire ses adieux éplorés aux différents membres de sa famille et à ses amis; et les chants ne prennent leur sens que par rapport aux étapes de ce parcours.

Même lorsqu'il n'y a pas de déplacement de l'énonciateur suivant un itinéraire programmé, beaucoup de chants cérémoniels (tels que, par exemple, les chants qui accompagnent la fête de dation du nom à un enfant [dénsagali dònkiri], les chants d'excision [kìne kìne dònkiri], les chants célébrant la fin des études coraniques [kàlanjigi ḋ̀nkiri], les chants funéraires des chasseurs [dàndaga d̀̀nkiri]) obéissent à un rituel fixe qui détermine leur succession. Et c'est de la relation dialectique entre les différentes phases de ce rituel et l'énoncé des chants produits à cette occasion qu'émerge le sens du procès énonciatif proprement dit.

Il va donc de soi que dans le cadre de performances régies par un rituel, l'objet d'étude pertinent ne doit pas être chaque œuvre prise individuellement mais la séance telle qu'elle s'est déroulée dans son ensemble. Les chants cérémoniels ne sont cependant pas les seuls à gagner à être pris en compte dans le contexte de la séance où ils ont été exécutés. Cette exigence s'impose aussi pour les genres agonistiques.

\section{Le cas des joutes verbales}

Chez les Dioula de Kong, comme en maintes sociétés, certains genres donnent lieu à des joutes verbales, en l'occurrence entre les hommes et les femmes. Chaque groupe interprète à tour de rôle, en puisant dans un répertoire consacré, de brèves épigrammes chantées à l'encontre du groupe opposé. Le principe du déroulement de la séance consiste donc, pour chaque camp, à trouver dans l'instant un chant du répertoire qui apparaisse comme une réplique du précédent. Ainsi en va-t-il de plusieurs types de chants donnés à l'occasion des cérémonies de mariage où jeunes gens des deux sexes s'échangent des provocations de façon ludique et conventionnelle [kónyon kúrun dònkiri et kónyon bèn dònkiri]. Voici, en traduction française, le début d'une séance de kónyon bèn dònkiri qui illustrera bien cette modalité de fonctionnement : 
1. Chant des hommes: Elles m'ont abusé,

Les femmes de ce pays m'ont abusé avec leur vagin.

$\begin{array}{ll}\text { 2. Chant desfemmes: } & \text { Ils m’ont abusée } \\ \text { Les garçons de ce pays m'ont abusée avec leur langue. }\end{array}$

Bawédié, bawédié, woyo waya!

3. Chant des hommes: Voilà le bruit de quelqu'un qui passe,

C'est le bruit que fait la vilaine fille en passant : woyo waya!

Bawédié, bawédié, woyo waya!

4. Chant desfemmes: Voilà le bruit de quelqu'un qui passe,

C'est le bruit que fait le vilain garçon en passant : woyo

5. Chant des hommes : C'est du plaisir pour nous tous, Je ne paierai pas. 6. Chant desfemmes: Si tu ne me donne pas d'argent,
Je ne coucherai pas avec toi.

Mon ami Basori, voici mon propos :

7. Chant des hommes: Les femmes de ce pays ont abandonné la quenouille Pour compter sur le commerce de leur cul.

Les hrorn aiment bien trouver un vieux champ abandonné,

8. Chant desfemmes: Les célibataires aiment bien les femmes d'aujourd'hui qui [sont libres d'elles-mêmes.

La femme est un singe noir,

9. Chant des hommes: C'est moi qui vous le dis.

À peine est-elle sur une branche,

Que c'est sur une autre qu'elle a envie d'aller, mon ami.

Ce qui me fait mal, c'est qu'on compare

10. Chant desfemmes: L'igname nouvelle avec le vieux manioc,

Qu'on les compare, voilà ce qui me fait mal. 
Dans cette séance, ce sont les hommes qui ont ouvert le feu et ce sont les femmes qui sont contraintes à la réplique. Il peut arriver aussi bien que ce soit l'inverse. Les chants fonctionnent donc d'abord par couples impairs-pairs, selon le principe attaque/contre-attaque : 1-2, 3-4, etc. À l'ouverture, alors que les partenaires de cette confrontation verbale ne se sont pas encore échauffés, on trouve des échanges faciles reposant sur un principe mécanique de symétrie inverse qui ne demande pas beaucoup de présence d'esprit, un chant appelant automatiquement son pendant inversé. Ainsi en va-t-il des quatre premiers chants : ces deux premières paires forment des couples indissociables dans ce jeu du tac au tac puisqu'il s'agit en réalité du même chant dont on a seulement inversé une brève séquence en changeant la fille par le garçon. On les retrouve couplés à l'initiale d'autres performances de kónyon bèn dìnkiri.

À partir du chant 5, dans lequel les hommes font mine de se révolter contre la vénalité des femmes en matière de commerce sexuel, l'enchaînement des partitions devient un peu plus subtil. Le chant 6 par lequel les femmes répliquent n'est plus en effet l'envers symétrique du chant des hommes ; c'est un chant autonome et sa fonction de réplique au chant 5 est davantage circonstancielle : les hommes, dans leur chant, dénoncent la vénalité des femmes, celles-ci la revendiquent. En outre, à partir de là, la partition des hommes va fonctionner elle-même comme une réplique à la partition des femmes qui était déjà une réplique à la provocation des hommes, si bien qu'une succession de couples pairs-impairs vient se superposer à celle des couples impairs-pairs et que chaque partition devient une réplique de la précédente aussi bien pour les femmes que pour les hommes. En effet, le chant 7 des hommes apparaît comme une réponse logique au chant 6 des femmes : dans celui-ci, elles ont affirmé leur droit à se faire payer pour faire l'amour, eh bien, nous dit le chant 7 , ce sont des putains, ce à quoi les femmes rétorquent aussitôt dans le chant 8 que les célibataires sont pourtant bien contents de trouver des femmes faciles. Le chant 9 des hommes rebondit sur ce thème de la liberté de mœurs pour stigmatiser la prétendue inconstance congénitale des femmes, à la suite de quoi celles-ci font mine de s'offusquer en suggérant dans le chant 10 que la valeur des femmes (l'igname nouvelle) est bien supérieure à celle des hommes (le vieux manioc). La suite de la séance, dont nous ne pouvons, dans les limites de cette contribution, donner l'intégralité, continue sur ce mode agonistique et ludique à coup de provocations stéréotypées : outre leur caractère volage, les femmes sont coquettes, dépensières, étourdies, les hommes sont avares, jaloux, prétentieux, ...

Cette fois encore, il est évident que c'est la structure de la séance et non chaque pièce particulière entrant dans sa composition qui constitue le fait culturel pertinent et qui révèle les clichés en cours dans la société en matière de représentation de l'autre sexe ainsi que la fonction cathartique de ce genre. 


\section{Le cas des genres circonstanciels}

Par cette dénomination, nous entendons d'abord des genres oraux dont la série d'œuvres (en l'occurrence des chants) interprétée au cours d'une séance est en relation directe avec une activité. La succession des chants rend alors compte du déroulement de cette activité et ceux-ci ne prennent sens que par rapport à elle. Ainsi sont chez les Dioula les chants agricoles [ṡ̀ncke ḋ̀nkiri], produits directement aux champs par des chanteurs tambourinaires, au moment des grands travaux agricoles collectifs où des cultivateurs de plusieurs familles se regroupent pour préparer à tour de rôle leurs champs respectifs. Ces chants ont pour fonction d'encourager et même de galvaniser les cultivateurs qui rivalisent d'ardeur entre eux pour butter par exemple leurs ignames ou leurs maniocs. Ainsi que va l'illustrer le début de la séance de sèncke dònkiri' qui suit, la succession des chants $^{3}$ va suivre au plus près le déroulement de l'activité laborieuse.

Chant $1 \quad$ Vais-je mourir au champ ?

Moi je n'en sais rien.

Le cultivateur dit : Hé, méchante souffrance

Assieds-toi et attends-moi.

Moi, je vais aller faire un tour. La souffrance répond : Hé, mon ami,

Chant 2 C'est que moi je ne te demande rien,

À toi cultivateur, je ne te demande rien.

La mauvaise friche est en brousse,

Les hommes se retrouvent en brousse, ohé !

Le tambour dit : avancez vite,

C'est l'intérêt du propriétaire du champ.

Chant 3 Si un lièvre est débusqué,

Il revient aux batteurs de tambour

Si un rat est débusqué,

Il revient aux batteurs de tambour.

2. Cette séance a été enregistrée en 1979 dans un champ appartenant à Tawakali Ouattara. 3. Nous ne donnerons ici, pour des questions de volume, que la phrase-clé de chaque chant qui, dans la réalité de son exécution, fait l'objet de nombreuses reprises avec quelques variantes. 
Hé Vali toi qui avances vite, reviens, arrive,

Chant 4

Que je proclame ton nom!

Toi qui retournes vite la terre, toi qui es rapide à faire les buttes,

Arrive ici, que, moi, je proclame ton nom !

Chant 5 Une bonne action est un bienfait

Est-ce qu'on ne doit pas en faire à l'égard de ceux qu'on connaît ?

$\begin{array}{cl}\text { Chant } 6 & \text { Oh, il est ici oh ! } \\ & \text { Hé, il est ici, hé, } \\ & \text { Oh, il est ici, oh! }\end{array}$

Dans une telle séquence qui débute une séance de sèneke dìnkiri, il apparaît de façon évidente que ces chants ne peuvent dans l'ensemble se comprendre que par référence au contexte situationnel immédiat de leur énonciation et que leur succession prend sens par rapport au déroulement évolutif de cette situation.

Le premier chant des tambourinaires est donné alors que les cultivateurs sont rassemblés dans le champ, prêts à entamer le travail collectif qui n'a pas encore commencé. La phrase-clé «Vais-je mourir au champ ? » qui est censée représenter l'interrogation angoissée que tous se posent secrètement avant de se mettre à l'œuvre, leur prête l'intention de «se tuer » à la tâche, d'aller au bout des limites de leurs forces, ce qui est une façon de les mettre effectivement dans cet état d'esprit. Par rapport à ce premier chant, le second, alors que le travail vient tout juste de commencer, évoque, dans sa première partie, la tentation de se dérober car cette tâche éreintante va causer beaucoup de souffrance. Mais la seconde partie du chant rappelle qu'il n'y a plus de dérobade possible, chacun est au pied du mur : les hommes sont rassemblés et le défrichage les attend. Le chant 3 les incite donc à ne pas lambiner et rappelle les règles du jeu de ce genre d'événement : au cours du défrichage, en particulier si on brûle des parcelles, il n'est pas rare de débusquer de petits animaux qui vivaient dans des terriers et qui sont brûlés par le feu. Ces prises sont le butin qui revient aux tambourinaires pour les remercier de l'aide qu'ils apportent aux travailleurs grâce à leurs chants qui leur donnent du cœur à l'ouvrage.

Le chant 4 est un chant qu'on va retrouver, avec quelques variantes une bonne vingtaine de fois au cours de la performance. Il consiste à féliciter un cultivateur qui, à un moment donné, s'est montré particulièrement vaillant et efficace. Dans le cas présent, il est adressé à un certain Vali, mais il suffit de changer le nom et 
les chanteurs interprètent ce chant à chaque fois qu'à leurs yeux quelqu'un s'est particulièrement distingué dans le maniement de la houe. Et tout au long de la performance, les chanteurs vont ainsi alterner les louanges individuelles qui peuvent prendre diverses formes (le chant 6 offre un exemple différent du chant 4 pour remplir la même fonction laudative) et les adresses plus générales destinées à motiver ou remotiver l'ensemble des travailleurs en leur rappelant, ainsi que le fait le chant 5 , les valeurs morales et sociales qui justifient l'effort qu'ils sont en train de faire par solidarité.

Il est bien évident que là encore c'est la structuration de la séance qui donne du sens à l'événement. C'est cette séance qui est le fait culturel pertinent, beaucoup plus que chaque chant pris en particulier, dont le signifié, coupé de son contexte, n'a d'ailleurs plus aucun intérêt, comme l'illustre bien le chant 6 . Que peut vouloir dire en termes de fonction culturelle un énoncé qui consiste à répéter l'exclamation «Oh, il est ici ! » ? On n'en comprendra la signification qu'en découvrant qu'il s'agit d'une louange récurrente adressée à tour de rôle à tel ou tel individu par le groupe des batteurs chanteurs qui est venu alors l'entourer pour le féliciter de son acharnement à la tâche. Et, bien sûr, pour le bénéficiaire de la louange, le fait d'être ainsi momentanément placé sur le devant de la scène est une incitation à redoubler d'ardeur.

\section{La séance, acte de création collective}

Jusqu'ici ont été envisagés des cas particuliers où des séries d'œuvres entrant dans la composition d'une performance étaient liées à un rituel, à une règle du jeu ou à une activité qui donnait sens à leur succession et aboutissait finalement à un métadiscours global cohérent. Mais ce supplément d'intérêt que représente la prise en compte d'une séance dans son ensemble existe aussi dans le cas de genres dont la succession au cours d'une performance est en apparence plus aléatoire et dépend de l'initiative des participants, indépendamment de toute contrainte extérieure. Ainsi en est-il des contes. L'analyse d'une veillée de contes tenue en décembre 1974, où se trouvaient réunies quatre femmes entourées de quelques enfants dans une cour du kábila Barola (chez les Baro), va nous montrer que, pour ne pas être déterminée par des facteurs extérieurs, la succession des dix-neuf contes qui ont été interprétés ce soir-là n'est peut-être pas aussi arbitraire qu'on pourrait le penser au premier abord. 
Donnons tout d'abord un bref résumé de ces dix-neuf contes dans l'ordre où ils ont été exécutés ${ }^{4}$.

1. Tortue et Éléphant ont une querelle à propos d'une source où ils avaient coutume de boire tous deux et qu'Éléphant a décidé de boucher. S'ensuit un combat entre eux au cours duquel Éléphant avale Tortue. Mais une fois dans le ventre d'Éléphant, Tortue déchiquette ses intestins à l'aide d'un petit couteau qu'il avait et ressort par l'anus tandis que le cadavre d'Éléphant est la proie des vautours. (interprète : Mawa Kurubari, hóronn', femme de 30 ans, épouse de tisserand)

2. Trois prétendants d'une jeune fille partent en voyage à Kumassi (Ghana). Elle demande à chacun un cadeau. Au premier un médicament traditionnel [sipe], au second des souliers extraordinaires, au troisième un miroir extraordinaire. À leur retour les jeunes gens font route ensemble. Celui qui a acheté le miroir y voit que la jeune fille qu'ils courtisent est morte à Kong. Celui qui a les souliers merveilleux les donne au possesseur du sipe qui d'un seul pas arrive à Kong et ressuscite la fille à l'aide de son médicament. Lequel des trois doit-elle épouser ? (interprète : Matulen Baro, hóron, femme de 40 ans, coépouse de la précédente)

3. Une orpheline vit chez sa marâtre avec sa demi-sœur. La mère prend soin de sa fille et néglige l'orpheline. Lorsque cette dernière va faire sa lessive au marigot, le lion lui donne de la viande en la prévenant par un chant qu'il y aura du sable dedans. La fille remercie, lave la viande dans le marigot et la mange. Voyant qu'elle prospère plus qu'elle, la demi-sœur de l'orpheline décide à son tour d'aller au marigot. Mais lorsque le lion lui offre la viande par son chant, elle la refuse sous prétexte qu'il y a du sable, si bien que le lion l'attrape et la tue. Moralité : si tu fais du mal à l'enfant d'un proche cela retombe sur toi. (interprète : Masəgona Baro)

4. Une jeune fille veut aller rendre visite à son amant de l'autre côté du fleuve. Le crocodile lui propose de la faire traverser à condition qu'elle n'en dise

4. On retrouvera le texte intégral des contes de cette veillée dans Derive, 1987, tome 3 , p. 610-689.

5. La catégorie des hóron est celle des individus libres de naissance ; celle des wóloso, qui est celle des anciens captifs, chargés des versions grivoises de la littérature orale en général. 
rien. Elle promet et le crocodile la fait traverser dans les deux sens. À son retour, sa mère puis son père lui demandent comment elle a franchi le marigot. Elle ne trahit pas le secret, malgré la promesse de somptueux cadeaux. Lorsqu'elle revient chez son amant qui l'interroge à son tour, elle finit par tout révéler contre la promesse d'un peigne. Lorsqu'elle retourne pour traverser à nouveau le fleuve, un oiseau révèle sa trahison en chantant. La fille lui donne du grain pour qu'il interrompe son chant pendant qu'il picore. Au crocodile qui l'interroge, elle répond qu'elle n'a pas parlé et celui-ci la fait traverser de nouveau. Mais lorsqu'elle est sur l'autre rive, l'oiseau qui a fini de picorer, reprend son chant délateur. Le crocodile dit qu'il n'a pas compris et demande à l'oiseau de s'approcher. Celui-ci vient se poser sur sa gueule et le crocodile l'avale. Les déchets qu'il recrache sont devenus de l'écume. (interprète : Masıgona Baro, 50 ans, femme wóloso)

5. Une femme stérile obtient un enfant d'un rocher contre la promesse d'un sacrifice. Une fois l'enfant obtenu la femme oublie de faire le sacrifice. Devenu grand et roi, l'enfant passe à cheval sur le rocher qui le reprend et l'absorbe. C'est pourquoi, si Dieu exauce tes désirs tu ne dois pas oublier d'en payer le prix. (interprète : Masogəna Baro)

6. Une sœur cadette, très belle, doit se marier avant son aînée. Celle-ci, jalouse, la noie dans le marigot. Une amie de la cadette vient régulièrement au bord de la rivière et par son chant fait revenir la noyée quelque temps sur la rive pour converser avec elle. Un chasseur surprend le secret et les villageois viennent pour essayer de l'attraper, mais elle rentre définitivement dans le marigot dont elle devient un génie. C'est depuis ce temps que, si on doit marier la cadette avant l'aînée, on fait d'abord asseoir cette dernière sur le tabouret de mariage avant sa petite sœur. (interprète : Masəgəna Baro)

7. Un beau jeune homme dont la mère est une sorcière courtise une jeune fille. Un jour que celle-ci décide d'aller voir son amant la nuit, la mère va au devant d'elle et l'emprisonne au faîte d'un arbre pour la dévorer. La jeune fille prévient son amant par un chant. Celui-ci vient la délivrer et tue sa mère. C'est pourquoi, si une fille vient te rendre visite, il ne faut pas laisser ta mère l'accompagner. (interprète : Masogəna Baro)

8. Une jeune fille refusait tous les prétendants qu'on lui proposait. Un soir, un homme décide de lui faire peur en la poursuivant avec un brasero rougeoyant qu'il secoue derrière elle. Elle court pour se réfugier chez sa 
mère, puis chez son père, puis chez son grand-frère mais tous refusent de lui ouvrir. Elle se rend alors chez le mari qu'on lui a proposé. Ce dernier lui ouvre la porte et ils s'unissent. C'est depuis ce temps qu'on prend les maris qu'on nous donne. (interprète : Masıgona Baro)

9. Un roi avait plusieurs femmes. Alors que sa favorite n'avait pas d'enfant, il engrosse la mal-aimée en l'avertissant qu'il n'y a pas de place chez lui pour son enfant. Celle-ci va accoucher en brousse, mettant au monde deux jumeaux dont elle confie l'un à la panthère, l'autre au lion. Devenus grands, les jumeaux partent à la recherche de leur génitrice et interrogent par un chant toutes les femmes de la contrée pour qu'elles disent où ils ont été mis au monde. Vu leur beauté, toutes les femmes revendiquent cette maternité, mais aucune n'est capable de leur donner la bonne réponse, jusqu'à ce qu' ils arrivent enfin à la mal-aimée qui se fait reconnaître comme leur mère. Le roi les accueille comme ses fils et la mal-aimée prend la place des favorites. (interprète : Matulen Baro)

10. Un roi avait dans sa cour un baobab dont il avait interdit que quiconque coupe une seule feuille. Un jour, une femme enceinte en coupa une et le roi la tua, lui ouvrit le ventre et confia l'enfant à sa grand-mère maternelle qui l'élève sans rien lui dire. Devenu grand, l'enfant est un lutteur invincible et tous ceux qu'il terrasse lui disent : « voilà pourquoi je ne te dis rien ». Il interroge sa grand-mère sur le sens de cette phrase sibylline et elle lui conseille de menacer d'égorger celui qu'il aura vaincu s'il ne s'explique pas. Il apprend ainsi la vérité et va trancher le cou du roi dont il prend la place. (interprète : Mamagangan Baro, fille d'une douzaine d'années, hóron)

11. Un roi avait deux femmes, une favorite et une mal-aimée, qui avaient chacune une fille. Quand elles vont protéger les récoltes des oiseaux, la fille de la favorite chante un chant où elle demande au fétiche de faire mourir le roi tandis que la fille de la mal-aimée lui demande de l'épargner. Grâce à un chasseur, le roi l'apprend, chasse la mauvaise fille et sa mère et fait de la mère de la bonne fille sa favorite. (interprète : Mawa Kurubari)

12. À sa mort, une femme confie son fils à sa coépouse. Mauvaise, celle-ci cherche à empoisonner sa nourriture, puis l'eau de sa toilette, mais elle échoue chaque fois car le garçon a un chien qui a tout vu et qui va au devant de lui pour le prévenir par un chant, à son retour de brousse. Dépitée, la marâtre creuse une fosse hérissée de fièches qu'elle dissimule par une natte 
où elle enjoint au garçon de s'asseoir. Mais, toujours prévenu par son chien, celui-ci refuse et c'est le propre enfant de la femme qui rentre en trombe, va aussitôt s'asseoir sur la natte et meurt empalé. Si tu fais le mal, Dieu le fait retomber sur toi. (interprète : Mawa Kurubari)

13. Un roi avait un champ au bord duquel se trouvait une énorme chenille. Il avait trois enfants qu'il avait envoyés surveiller les champs. Les deux premiers saluent poliment Chenille mais le troisième salue sans respect et Chenille l'avale. Alors le roi envoie à tour de rôle tous ses enfants pour tuer Chenille mais au moment ou ils s'apprêtent à brandir leur lance, Chenille entame un chant qui les charme tant qu'ils ne peuvent faire autrement que danser, jusqu'à briser les jambes de leur cheval. Suite à ces essais infructueux, le roi décide d'aller combattre Chenille lui-même mais lui aussi est pris par le charme du chant et danse jusqu'à s'en briser le pied. Chenille est venue s'installer à la cour où elle est devenue chanteuse. (interprète : Mawa Kurubari)

14. Un homme avait deux femmes. Sa préférée est morte et il a tellement de chagrin qu'en pleurant il laisse sortir ses parties génitales que l'autre épouse est obligée de rentrer dans le pantalon ${ }^{6}$. (interprète : Masogona Baro)

15. Une femme, en mourant a laissé sa fille à sa coépouse. Alors que la fille de cette dernière est choyée, l'orpheline n'a que des plats sans sauce qu'elle va manger sur la tombe de sa mère tout en chantant son infortune. À la fin, Dieu prend pitié d'elle et ressuscite la mère. Si tu maltraites l'enfant de ta coépouse, le mal retombera sur toi. (interprète : Masogona Baro)

16. Autrefois, la lionne, la vache et l'ânesse élevaient leurs enfants en commun dans la brousse, mais un jour l'enfant de la vache casse le cou du lionceau. Quand elles s'en aperçoivent, la vache et l'ânesse fuient vers le village et la lionne qui découvre le drame plus tard se met à les poursuivre. La vache peut se cacher, mais la lionne a le temps de griffer l'ânesse à l'orée du village, d'où la traînée noire que les ânes ont à l'arrière-train. C'est depuis ce temps que les ânes et les vaches appartiennent au monde du village et les lions à celui de la brousse. (interprète : Matulen Baro)

6. Ce conte paillard émaillé de termes grossiers est donné par une descendante de captifs [wóloso]. Il est dans la tradition des contes qui sont dévolus à cette catégorie sociale. 
17. Autrefois le lion et l'enfant d'homme pêchaient ensemble. Mais un jour la mère du garçon s'impose à la pêche malgré les réticences de son fils. Ce dernier décide de la faire grimper sur un arbre pour la protéger, mais elle urine puis défèque sur le lion et finit par tomber de l'arbre. Le lion la prend dans sa besace et sur le chemin du retour l'enfant déplore dans un chant la perte de sa mère. Le lion lui demande chaque fois ce qu'il dit et l'enfant répond qu'il se plaint d'avoir laissé tomber son poisson. Finalement, le lion décide d'aller chercher un récipient pour mettre le poisson du garçon. Dès que le lion est parti, l'enfant délivre sa mère et ils se sauvent vers le village. À son retour, le lion les poursuit, mais ils ont le temps d'arriver. Alors le lion proclame la fin de l'alliance avec l'enfant. Tous les humains resteront au village et lui, qui ne pêchera jamais plus avec les hommes, restera en brousse. (interprète : Matulen Baro)

18. Une femme a accouché d'une enfant-crapaud. Quand elle part vaquer à ses occupations, elle donne du mil à piler à sa fille, mais celle-ci ne fait que chanter et uriner dedans, si bien que la femme retrouve le mil comme elle l'avait laissé et doit le piler elle-même pour préparer le repas. Or, un jour, arrive une autre fille qui pile le mil à la place de la fille-crapaud. La femme pense que c'est sa fille qui s'est enfin décidée à travailler et pour en avoir le cœur net décide de la guetter. Elle voit l'enfant inconnue arriver, piler le mil et le cuisiner. Elle pense que c'est sa fille-crapaud qui se transforme ainsi en humain et elle décide de la garder avec elle. Mais la mère de la fille humaine vient la réclamer. Elles se disputent alors la propriété de l'enfant et la mère de la fille-crapaud, voyant que l'autre ne voulait pas lui laisser la fille humaine, finit par la tuer. Alors sa fille-crapaud qui n'était pas loin réapparaît. Dépitée sa mère la jette au loin, mais elle revient et la mère est obligée de la garder. Quand tu as un enfant, quel qu'il devienne, tu ne peux jamais le renier. (interprète : Mawa Kurubari)

19. Une femme meurt en laissant un orphelin qu'on confie à sa grand-mère. Cet enfant était un fruit de fromager que Dieu avait transformé en garçon sur les instances de la mère, stérile. Elle l'avait appelé Mamori Barro et quand son repas était prêt, elle l'appelait par son nom pour qu'il vienne manger. Mais la grand-mère, lorsqu'elle le hèle pour le repas l'appelle «Enfant du fromager ». Malgré les pleurs de l'enfant et les remontrances du père, elle réitère son comportement plusieurs fois, si bien que l'enfant finit par retourner à son état premier de fruit du fromager. (interprète : Mawa Kurubari) 
Pour montrer comment la structuration de cette séance de contes aboutit elle-même à un discours cohérent de second degré, deux grands types de traits sont à retenir :

- d'une part, le thème et le prédicat des contes. En effet dans la mesure où ces récits ont un contenu idéologique marqué par rapport à la morale sociale, la nature du discours global mis au jour par l'enchaînement des contes au cours de la soirée permet de penser le contenu d'ensemble de la séance comme œuvre collective au deuxième degré, révélatrice de points de vue dominants.

- d'autre part, la structure des contes. Leur enchaînement dans la séance, la proportion de différents types morphologiques de récits représentés (ascendant/descendant, cyclique, positif/négatif, en miroir, en sablier ${ }^{7}$ ) peuvent donner des indications significatives sur la philosophie de la société, selon qu'elle est plus ou moins conservatrice ou progressiste. Ces traits peuvent aussi témoigner d'un certain souci esthétique par une composition équilibrée.

$\mathrm{Du}$ point de vue thématique, deux grands ensembles peuvent être tout d'abord dégagés de la production de cette séance. L'un traite essentiellement des relations d'alliance matrimoniale $(2,6,7,8,14)$, l'autre des relations entre parents et enfants, avec un fort accent sur la relation maternelle (n'oublions pas que ce sont des femmes qui content). Ce second ensemble englobe tous les autres

\footnotetext{
7. Cette typologie, aujourd'hui bien connue et faisant autorité, vient d'un article de Denise Paulme (1972) qui a adapté au conte africain les catégories morphologiques de Propp en étudiant les combinaisons possibles de quelques fonctions fondamentales : manque, quête, épreuve, situation d'euphorie ou de dysphorie. Pour mémoire, le type ascendant comprend au moins trois fonctions centrales se succédant dans cet ordre : manque (ou persécution) - amélioration - manque comblé (ou persécution abolie); le type descendant suit un trajet inverse : situation d'équilibre - détérioration - manque ; le type cyclique, après une série d'améliorations et/ou de détériorations, présente un trajet qui ramène à l'état initial. Il sera dit positif ou négatif, selon que cet état initial était euphorique ou dysphorique ; le type en miroir présente successivement deux héros, le premier positif, le second négatif qui entreprennent deux quêtes symétriques aux résultats opposés; le type en sablier présente deux héros se trouvant au départ dans des situations opposées (euphorie/dysphorie) qui vont échanger leurs positions respectives au cours d'un trajet symétriquement inverse. Denise Paulme distingue encore d'autres types dont nous ne faisons pas état ici dans la mesure où ils ne sont pas représentés dans les contes de cette séance.
} 
contes, à l'exception du premier qui, traitant du motif de la victoire du plus faible sur le plus fort, est le seul marginal par rapport à ces deux axes thématiques, sans pour autant être dépourvu de pertinence à l'initiale de la chaîne quand on sait que les femmes, largement dominées par le pouvoir mâle, sont précisément perçues comme le sexe faible. Il faut aussi signaler le cas un peu particulier du conte 4 qui, lui, est à cheval sur les deux ensembles dans la mesure où il traite de la rivalité entre la relation parentale et la relation matrimoniale.

Considérons maintenant les prédicats de chacun de ces deux ensembles thématiques. Presque tous les contes se rapportant aux relations d'alliance matrimoniale traitent du problème de la conquête de l'époux ou de l'épouse (2, $6,7,8)$. Loin de produire un discours répétitif, ils se complètent et se répondent les uns aux autres. Les deux premiers (2 et 6 ) abordent le thème de la rivalité dans cette conquête. Dans le premier cas, nous avons trois hommes en situation de rivalité positive (puisqu'ils coopèrent pour ressusciter leur bien-aimée) et qu'il est entendu qu'elle épousera l'un des trois ${ }^{8}$ (type ascendant). Dans le second, deux sœurs sont en relation de rivalité négative, puisque l'aînée noie la cadette qu'on se prépare à marier avant elle et que ce méfait est irréversible (type descendant). Il est facile d'apercevoir une série d'oppositions terme à terme entre ces deux discours symétriques :

Hommes en quête d'une épouse versus femmes en quête d'un époux Rivalité bien comprise succès versus rivalité mal comprise échec

Le conte 6 apparaît donc comme le pendant du conte 2 donné par Matulen Baro.

Les contes 7 et 8 quant à eux évoquent les autres dangers qui peuvent menacer la conclusion d'une bonne alliance matrimoniale et, cette fois encore, ils sont de nature inverse. Le premier (la mère du jeune homme est une sorcière qui attend l'amante de son fils pour la dévorer), traite, sous forme symbolique, du danger d'assimilation totale de l'épouse par sa belle-famille (la tentative de dévoration est à ce propos significative au plan symbolique). C'est le futur mari qui doit veiller à éviter cet excès risquant de compromettre l'équilibre de l'alliance, en « tuant », psychanalytiquement parlant, sa mère dévorante, autre expression symbolique de la rupture du cordon ombilical, rupture rendue nécessaire par le nouvel état qu'il se prépare à prendre. Un tel point de vue insiste donc sur le fait que la femme, en entrant chez son mari, n'embrasse pas une nouvelle famille. Elle

8. La réponse est donnée par l'auditoire, mais c'est toujours le plus jeune (celui qui lui administre le médicament miracle qui est choisi. Voir à ce propos DerIVE, 1976). 
garde son identité et continue à appartenir à son clan d'origine : le mariage est une alliance et non une assimilation.

Dans le conte 8 , le danger qui compromet l'alliance provient de la situation inverse : une identification telle de la jeune fille à sa propre famille qu'elle lui fait exclure toute possibilité d'alliance avec d'autres (elle refuse tous les prétendants). L'équilibre de la société se trouve donc menacé d'une autre façon (plus d'échange possible de femmes) et il ne sera rétabli que grâce à un subterfuge qui permettra de modifier l'attitude de la fille en l'amenant à accepter un mari. La présence conjointe de ces deux contes de structure cyclique dans la séance (les deux tentations d'assimilation, à sa belle-famille ou à sa propre famille, sont conjurées) nous fait donc comprendre qu'une alliance bien comprise doit se conclure entre ces deux écueils.

Le dernier conte relatif au thème de l'alliance matrimoniale (14) doit être considéré avec beaucoup de précaution car il représente un cas à part : il s'agit d'un conte wóloso $^{9}$ (groupe auquel appartient l'interprète Masəgəna Baro), donc, par principe, d'un discours parodique, sensiblement plus court que les autres dont la fonction est surtout de détendre l'auditoire. Toutefois, le choix spécifique de son thème, au sein du répertoire wóloso, n'est pas innocent dans le contexte de cette séance. Il s'agit d'un époux qui est tellement secoué de sanglots à la mort de sa femme préférée que ses parties génitales en sortent de ses vêtements. C'est son autre épouse qui doit les lui rentrer. Nous sommes certes en présence d'un thème burlesque; il n'empêche que pour terminer le discours global relatif au thème de l'alliance matrimoniale, ce dernier conte répond à l'ensemble qui le précède. Celui-ci traitait du problème de la conquête de l'épouse, cette fois c'est le problème de sa perte qui est abordé. Il est rappelé une fois de plus que, dans une société polygame, le mariage n'est qu'une alliance si bien que l'attachement au conjoint doit avoir des limites, faute de quoi tous les désordres risquent d'arriver. Et ce n'est sans doute pas par hasard si c'est la coépouse de la défunte qui se charge de rétablir la situation en remettant en place le sexe du mari. Même si l'homme a une femme préférée, toutes ses épouses lui sont utiles.

Passons maintenant à l'examen du second ensemble thématique, le plus important en volume, puisque avec douze contes, il englobe presque les deux tiers de l'échantillon produit au cours de la soirée. Neuf d'entre eux concernent plus spécialement la relation maternelle.

9. C'est-à-dire d'un conte de captifs. Il n'y a évidemment plus de captifs à Kong aujourd'hui mais leurs descendants continuent d'appartenir à une catégorie sociale spécifique, dont le propre, au plan de la culture verbale (contes, chants,...), est d'énoncer des grossièretés paillardes. 
Dans ce groupe, quatre contes $(9,10,11,17)$ présentent cette relation sous un jour positif : l'enfant y apparaît comme quelqu'un dont les qualités valorisent sa génitrice, compensant largement les déboires qu'elle a pu connaître par ailleurs. Dans le conte 9, la mal-aimée, grâce à ses jumeaux qui la retrouvent, voit son statut se transformer en celui d'épouse choyée. Il en va de même dans le conte 11 où, grâce aux qualités de son enfant, la mère de la bonne fille inverse sa situation avec la mère de la mauvaise fille qui était jusque-là la préférée. Dans le conte 10, le fils, devenu grand, venge sa mère, autrefois mise à mort par le roi, en le tuant à son tour et en prenant sa place. Enfin, dans le conte 17, l'enfant sauve sa mère prisonnière de son compagnon de pêche, le lion, donnant ainsi la primauté à son lien filial sur une autre relation sociale fondée sur la coopération économique, qui se trouve du coup détruite.

En contrepoint à cette vision positive de la relation maternelle (le comportement positif étant d'ailleurs toujours le fait de l'enfant, la mère n'étant que le bénéficiaire), trois autres contes $(3,12,15)$ la présentent sous un jour défavorable, sur le thème de l'orphelin maltraité par la coépouse de la défunte (le comportement négatif étant cette fois du côté de la marâtre qui joue le rôle de la mauvaise mère).

Dans ces deux sous-ensembles, ce ne sont pas deux discours inverses qui sont tenus sur la même réalité, mais une même vision idéologique appliquée à deux réalités opposées :

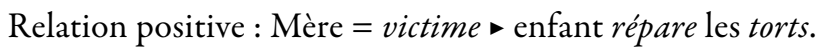

Relation négative : Marâtre = bourreau $\bullet$ Enfant punit les méfaits (avec l'aide d'alliés)

L'enfant est donc toujours un héros positif et il est régulièrement gagnant à la fin des contes. C'est ce qui explique que tous ces récits aient une structure à tendance ascendante, puisque, outre des contes proprement ascendants, on a des contes en miroir ou en sablier où les méchants sont punis et les bons récompensés. Une seule exception avec le conte 17 où, pour sauver sa mère, l'enfant perd le bénéfice de sa coopération avec le lion. Cela tient au fait que dans ce conte, à la différence des autres, la situation de victime de cette dernière n'est pas un état initial, mais la conséquence d'une faute de sa part entraînant logiquement une détérioration de la situation.

Restent encore deux autres contes de cet ensemble traitant de la relation maternelle $(5,18)$. On peut les regrouper à leur tour dans un sous-ensemble car eux aussi traitent un même sujet en relation symétrique inverse. Ces deux récits abordent le problème du désir de maternité. 


\begin{tabular}{|c|c|c|c|c|c|c|c|}
\hline Conte 5: & 1 & 2 & & 3 & 4 & & 5 \\
\hline & $\begin{array}{c}\text { Une femme } \\
\text { stérile }\end{array}$ & désire & & $\begin{array}{l}\text { nfant } \\
\text { 'elle } \\
\text { ient } \\
\text { ocher } \\
\text { açon } \\
\text { turelle. }\end{array}$ & $\begin{array}{c}\text { Elle refuse } \\
\text { inconsciem- } \\
\text { ment cette } \\
\text { origine } \\
\text { surnaturelle } \\
\text { (oubli } \\
\text { significatif } \\
\text { du sacrifice } \\
\text { promis) }\end{array}$ & & $\begin{array}{l}\text { e ne peut } \\
\text { erver son } \\
\text { fant. }\end{array}$ \\
\hline Conte 18 : & 1 & 3 & & 2 & 4 & & 5 \\
\hline & $\begin{array}{c}\text { Une femme } \\
\text { (féconde) } \\
\text { engendre }\end{array}$ & $\begin{array}{c}\text { defay } \\
\text { natur } \\
\text { une en } \\
\text {-mon }\end{array}$ & $\begin{array}{l}\text { on } \\
\text { ale } \\
\text { ant } \\
\text { tre }\end{array}$ & $\begin{array}{l}\text { qu'elle } \\
\text { rejette. }\end{array}$ & $\begin{array}{r}\text { Elle ref } \\
\text { d'accepter } \\
\text { donnée nat } \\
\text { (elle croit } \\
\text { facilement } \\
\text { métamorp } \\
\text { de la fille } \\
\text { paud en la } \\
\text { idéale) ma } \\
\text { peut }\end{array}$ & $\begin{array}{l}\text { tte } \\
\text { elle } \\
\text { op } \\
\text { la } \\
\text { ose } \\
\text { a- } \\
\text { lle } \\
\text { ne }\end{array}$ & $\begin{array}{c}\text { renier } \\
\text { son } \\
\text { enfant. }\end{array}$ \\
\hline
\end{tabular}

La numérotation des termes en italiques montre bien les oppositions terme à terme. La leçon de cet ensemble est claire : une femme ne peut s'approprier d'autres enfants que les siens (c'est-à-dire ceux qu'elle a elle-même engendrés ou ceux de sa coépouse qui, chez les Dioula, doivent être considérés comme ses propres enfants) et toute adoption de l'extérieur est précaire et toujours menacée (conte 5). À l'inverse, la mère doit accepter ses propres enfants tels qu'ils sont, avec leurs défauts, et non rêver l'enfant idéal (conte 18).

La relation maternelle est donc présentée comme un lien biologique (indéfectible) et non comme un lien électif (qu'on peut défaire à volonté). Dans les deux cas, nous sommes en présence de contes cycliques négatifs puisque la fin nous ramène à un état initial qui était dysphorique : désir non comblé d'enfant (conte 5), affliction d'avoir une enfant-crapaud (conte 18). Comme tels, ces contes s'opposent donc à la valeur des sept autres contes qui forment la classe consacrée à la relation maternelle. À lui seul, le conte 18 , avec une enfant 
monstrueuse dont le comportement négatif (la fille-crapaud urine dans le mil qu'on lui demande de piler) fait honte à la mère au lieu de la valoriser, s'oppose plus particulièrement au groupe des quatre contes $(9,10,11,17)$ qui présentait l'enfant comme un faire-valoir pour la mère.

Les trois derniers contes de ce grand ensemble de douze récits traitant de la relation parentale ne sont plus spécifiquement centrés sur le lien maternel. Deux d'entre eux forment encore un couple plus ou moins symétrique (13 et 19). Le conte 13, relatant l'histoire de l'enfant effronté avalé par Chenille, met en scène le père qui cherche, en vain, à venger son fils. L'intervention du père et son échec final laissent entendre que c'est lui qui, au sein de la famille, a la responsabilité de la bonne éducation sociale de ses enfants. Il perd irrémédiablement l'un des siens, parce que, à la différence des autres, celui-ci n'a pas respecté le code de politesse pour saluer Chenille. Dans le conte 19, où on retrouve le motif de l'enfant surnaturel qui disparaît, retrouvant son état initial de fruit du fromager, l'effronterie et le manque de respect des conventions sont cette fois le fait des parents, avec le comportement transgressif de la grand-mère, qui appelle l'orphelin « fruit du fromager ». Si l'effronterie des enfants est donc un facteur de déséquilibre social, il en va de même du manque de respect des parents et il est possible d'établir l'équation suivante :

\begin{tabular}{|l|c|c|c|c|}
\hline Conte 13: & Enfant & disparaît & par sa faute & à cause de son effronterie. \\
\hline Conte 19: & Enfant & disparaît & $\begin{array}{c}\text { par la faute de sa } \\
\text { grand-mère }\end{array}$ & $\begin{array}{c}\text { à cause de l'effronterie de } \\
\text { celle-ci. }\end{array}$ \\
\hline
\end{tabular}

Le troisième conte traitant de cette relation parentale élargie (16) présente une sorte de mythe pour justifier l'existence de la cellule familiale : autrefois, les parents (représentés par des animaux, ce qui est souvent le cas dans les récits à forte charge mythique) élevaient collectivement leurs enfants. Mais, à la suite de querelles entre ces derniers, les parents ont dû à leur tour se séparer pour former des familles distinctes. C'est donc l'enfant (qui se trouve au centre de tous les récits composant ce deuxième ensemble) qui est présenté comme le fondement de la cellule familiale de base.

Reste à examiner le conte 4 qui est à cheval sur le groupe des récits traitant de l'alliance matrimoniale et sur celui des récits traitant du lien parental. Il aborde en effet le problème de la rivalité entre ces deux types de relation : la jeune fille, tenue au secret par son pacte avec le crocodile, refuse de le révéler à ses parents mais finit par céder à son bien-aimé. Bien sûr, à un premier niveau d'interprétation, élémentaire, elle le fait par convoitise, voulant absolument 
74 Geneviève Calame-Griaule - n 83

obtenir le peigne qu'elle voit chez son amant. Il n'empêche qu'elle n'est finalement pas punie d'avoir trahi de cette façon son pacte et que c'est l'oiseau seul qui est châtié comme délateur trop zélé. Il est donc possible de se demander si, à un autre niveau, le conte n'affirme pas, sous une forme imagée, la primauté de la relation d'alliance sur la relation familiale : une fois mariée, la femme doit avoir plus d'intimité avec son mari qu'avec ses parents. Le fleuve traversé avec l'aide du crocodile est l'expression métaphorique de ce changement d'état.

En ce sens, le conte 4 forme une trilogie avec la paire déjà identifiée des contes 7 et 8 qui évoquaient les deux dangers susceptibles de menacer la relation d'alliance : l'assimilation excessive à sa belle-famille ou à sa propre famille. Si la solution est l'équilibre entre ces deux excès, la femme, lorsqu'elle arrive en âge d'être mariée, peut légitimement prendre de la distance par rapport à ses parents (elle ne leur livre pas le secret malgré leur insistance) et construire une relation d'intimité plus étroite avec son futur mari.

Il est remarquable d'ailleurs que ces trois contes se trouvent tous dans la première intervention en série de Masogəna Baro, ce qui montre la cohérence interne du discours propre à cette conteuse. En effet, le problème de la composition de la séance peut se poser à deux niveaux. Dans la mesure où la veillée de contes est un ensemble de productions individuelles qui se succèdent une à une ou par séries plus ou moins longues, il est possible, d'une part d'étudier la cohérence interne du répertoire produit au cours de la soirée par chaque conteur ou conteuse, d'autre part d'étudier la cohérence entre les discours des différents interprètes. Dans la séquence présentement analysée, une cohérence existe à chacun de ces deux niveaux : les conteuses suivent un fil discursif à la fois par rapport à leur propre production et par rapport à celle des autres.

L'équilibre d'une telle composition où se trouvent évoqués les différents aspects du thème central de la séance (relations familiales + relations d'alliance ${ }^{10}$ ) et les différents points de vue selon lesquels on peut les envisager, montre que la succession des œuvres, par tout un réseau complexe de relations de convergence ou de symétrie inverse, n'est pas distribué au hasard. Les prédicats variés des conteuses qui se répondent les uns aux autres sur une même question aboutissent au final à un discours de sagesse populaire dialectique et nuancé. Les interprètes ont donc participé à un acte de création collective en produisant non seulement une série de contes, mais en élaborant, avec la veillée même, une œuvre de degré supérieur à laquelle elles ont su donner une forme achevée. Le cas particulier de

10. Seul, le premier conte de la soirée, qui sert d'ouverture à la veillée, reste marginal par rapport à ce thème central. 
cette veillée de contes n'est qu'un exemple pour mettre au jour ce phénomène, mais il est valable pour toutes les séances de contes ou de devinettes ${ }^{11}$.

\section{Conclusion}

Les diverses illustrations présentées ici ont montré que, dans tous les cas où une performance de littérature orale donnait lieu à une production d'œuvres en série (chants, récits, etc.), il était pertinent de considérer l'intégralité de cette performance comme une œuvre en elle-même à un autre niveau, performance dont la structuration spécifique était susceptible de produire un supplément de sens par rapport à celui qui peut être dégagé de chacun de ses items particuliers. Cela montre aussi que les interprètes de l'oralité ne sont pas des simples machines à transmettre un patrimoine. Leur part de créativité n'est pas négligeable par le choix qu'ils font d'introduire telle ou telle œuvre à un moment donné de la performance, compte tenu du contexte verbal en amont; ainsi que par le traitement qu'ils font de cette œuvre en fonction de ce contexte particulier. C'est pourquoi il est important de recueillir autant que possible les productions de la culture orale dans des conditions naturelles et de les analyser non seulement dans leur contexte social mais aussi dans leur contexte énonciatif. Cette démarche ethnolinguistique reste encore trop rare et gagnerait à se généraliser pour toutes les études de littérature orale.

\section{Bibliographie}

Calame-Griaule Geneviève, 1970, « Pour une étude ethnolinguistique des littératures orales africaines » in POTTIER Bernard (dir.), Langages, $\mathrm{n}^{\circ} 18$, p. 22-47, DOI : $10.3406 /$ lgge.1970.2026.

Calame-Griaule Geneviève, 1977, Langage et cultures africaines : essais d'ethnolinguistique, Maspero, Paris, 364 p.

Calame-Griaule Geneviève, 2001, «Un itinéraire en ethnolinguistique » in Cabiers de littérature orale, $\mathrm{n}^{\circ} 50$, p. 15-33.

11. Nous avons d'ailleurs montré par ailleurs qu'une séance de devinettes faisait aussi, de par ses enchaînements, l'objet d'une création collective cohérente. DerIVE, 1980. 
Derive Jean, 1976, « Les trois prétendants rivaux en Europe et en Afrique » in Cabiers de littérature orale, $\mathrm{n}^{\circ} 1, \mathrm{p} .67-94$.

Derive Jean, 1980, « La maison éclatée, devinette dioula de Kong » in Dumestre Gérard (dir.), Recueil de littérature manding, ACCT, Paris, p. 182-214.

Derive Jean, 1987, Fonctionnement sociologique de la littérature orale : l'exemple des Dioula de Kong (Côte d'Ivoire), 3 volumes, Institut d'Ethnologie (coll. Sciences Humaines, série « archives et documents »), Paris, 2326 p.

Paulme Denise, 1972, « Morphologie du conte africain. » in Cahiers d'études africaines, $\mathrm{n}^{\circ} 45$, vol. 12, p. 131-163, DOI : 10.3406/cea.1972.2775.

Résumé : cet article s'attache à montrer que, dans la perspective d'une approche ethnolinguistique telle que Geneviève Calame-Griaule a pu la prôner, la séance, entendue comme une unité de «performance », peut être un niveau particulièrement pertinent d'analyse, dans le cas où y sont exécutées par des interprètes différents des séries d'œuvres (contes, chants, devinettes, etc.) dont la succession est souvent loin d'être arbitraire mais obéit à des logiques, déterminées par différents types de facteurs. La mise au jour de ces logiques est souvent capitale pour saisir la fonction anthropologique d'un événement énonciatif dans une culture donnée. En effet, si chaque unité est porteuse d'une signification spécifique (la morale d'un conte par exemple), sa place dans le déroulement d'une chaîne énonciative participe aussi de la construction d'un sens culturel global propre à la séance par un mécanisme complexe d'analogies et d'oppositions dialectiques. Le propos est illustré par un certain nombre d'exemples de séances empruntés à la littérature orale des Dioula de Kong (Côte d'Ivoire) qui illustrent différents cas de figure emblématiques : séances de chants rituels et cérémoniels, séance de chants corporatifs, séance de contes.

Mots-clefs : Dioula, Kong, Côte d'Ivoire, analogie, chant, conte, interprète, performance, séance. 


\section{The Collective Performance as a Relevant Level for Studying Oral Literature}

Abstract: this paper intends to show that, according the way of an ethnolinguistic approach, such as those initiated by Genevieve Calame-Griaule, the whole of a "performance", is a relevant level for the analysis of a cultural discourse, in the case of performances offering a succession of literary works (tales, songs, riddles...) acted by different interpreters. This succession indeed, far from being arbitrary, is generally governed by logical processes, according to different data. The study of these processes is often important to understand the anthropological significance of an enonciative event in a society. If each unit indeed has a specific meaning (the morality of a tale for example), its insertion at a particular place in the enonciative chain of the performance participates also in the construction of a global cultural significance by the mean of a complex mecanism of analogies and dialectic oppositions. The analysis is illustrated by several examples issued of Dyula Oral literature (Kong, Ivory Coast): performances of different kinds of songs and tales.

Keywords: Dyula, Kong, Ivory Coast, analogy, song, tale, interpreter, performance.

\section{Note sur l'auteur}

Jean Derive est professeur émérite de l'université de Savoie et membre du LLACAN. Il a soutenu sa thèse d'État sous la direction de Geneviève Calame-Griaule en 1987 : Le Fonctionnement sociologique de la littérature orale : l'exemple des Dioula de Kong (Côte d'Ivoire). Entre autres travaux, il a consacré de nombreuses publications aux genres de la littérature orale de cette société ainsi qu'à la théorie de la littérature orale. 